\title{
Exploring the use of robots as social mediators in a remote human-human collaborative communication experiment
}

\author{
Fotios Papadopoulos*, Kerstin \\ Dautenhahn $^{\dagger}$, Wan Ching $\mathrm{Ho}^{\ddagger}$ \\ University of Hertfordshire, \\ School of Computer Science, UK \\ Received 2012/01/13 \\ Accepted 2012/04/20

\section{Abstract} \\ Our long-term goal is to develop robots as social mediators that can support human-human communication in remote \\ interaction scenarios. This paper explores the effects of an autonomous robot on human-human remote communi- \\ cation and studies participants' preferences in comparison with a communication system not involving robots. We \\ developed a platform for remote human-human communication in the context of a collaborative computer game. The \\ exploratory study involved twenty pairs of participants who communicated using video conference software. Partic- \\ ipants expressed more social cues when using the robot and sharing of their game experiences with each other. \\ However, analyses of the interactions of the participants with each other and with the robot show that it is difficult for \\ participants to familiarise themselves quickly with the robot while they can perform the same task more efficiently with \\ conventional devices. These issues need to be carefully considered and addressed when designing human-human \\ remote communication systems with robots as social mediators. \\ Keywords \\ $H R I \cdot$ collaborative game $\cdot$ remote communication $\cdot$ social mediation $\cdot$ computer game $\cdot$ robot synchronisation
}

1. Introduction

Nowadays, robots can be found in domestic environments performing various types of tasks, such as entertaining the users (e.g. Sony AlBO', $\mathrm{NAO}^{2}, \mathrm{PLEO}^{3}$ ) or assisting the users with a physical task such as carrying heavy objects within the house (Pioneer robots ${ }^{4}$ ) or even with tasks like vacuum cleaning the house $\left(R_{0} o m b a^{5}\right)$. As technology improves day by day, robots will become far more flexible, making them able to perform more complicated tasks and communicate with the users in a more natural and sociable manner. The European LIREC project (LivIng with Robots and IntEractive Companions) ${ }^{6}$ is a collaboration of 10 European partners specialised in psychology, ethology, human-computer interaction, human-robot interaction, robotics and graphical characters and aims to create a new generation of interactive and emotionally intelligent companions that are capable of establishing long-term relationships with humans. In this project, the research group is developing methods that allow a companion robot to perceive, remember and react to human users, thus enabling the companions to be socially aware in a domestic environment.

\footnotetext{
E-mail: f.papadopoulos1@herts.ac.uk

${ }^{\dagger}$ E-mail: k.dautenhahn@herts.ac.uk

¥E-mail: w.c.ho@herts.ac.uk

1 http://www.sony.co.uk/support/en/hub/ERS

2 http://www.aldebaran-robotics.com/

3 http://www.robotsrule.com/html/pleo_robot.php

4 http://www.mobilerobots.com/researchrobots/

pioneerp3dx.aspx

5 http: //www.irobot.com/uk/store.aspx

6 www.lirec.eu
}

The LIREC project comprises different human-robot everyday interaction scenarios. At the University of Hertfordshire we investigate the role of robots in the domain of physical assistance (e.g. fetch-and-carry tasks) and cognitive prosthetics (i.e. reminding a user of appointments). Additionally, we explore the role of interactive robots as communication mediators for enhancing the distanced communication between humans by utilising collaborative tasks. The development of a robot as a social mediator provides the context of the research reported in this article.

It is not uncommon that people occasionally or permanently live away from their friends and families and communicate with each other mediated by electronic interfaces. Often, in particular for longer distances, people utilise video communication software that provides richer experiences. However even these technologies, traditionally utilising audio and video, lack one very important medium of interaction which is tactile sensation (i.e. touch). Increasingly researchers have been studying how touch could be transferred over the communication channel to enhance the users' experience and provide more interactive and enjoyable communication. In the context of remote communication, and especially in our experiment, enhancement of user experience is defined as an increase of social cues, tactile interactions and non-task related speech expressed by the users. These measures are quantifiable and can highlight differences in comparative studies investigating tactile and non-tactile interfaces. Remote communication technology might include a device worn by the user (e.g. tactile gloves [1]) or robots in various forms and shapes which have been used for domestic entertainment purposes with some of them offering advanced social interactions with humans [2]. In order to explore the role of robots on distanced communication, we developed a novel computer application named "AiBone" to socially connect the participants and undertake the communication between the robots. The overall goal of our research is to develop robots as social mediators that can support human-human communication in remote interaction scenarios such as the social me- 
diator scenario in the LIREC project where two distanced users wish to communicate by utilising their personal robot companions. This article describes the development of the system as well as the evaluation with pairs of users.

AiBone is a remote communication system that aims to enable two participants to play individually with their autonomously operating AIBO robot while the users simultaneously cooperate with each other in an interactive collaborative task through existing video communication software by utilising the robots as mediators of the interaction. The AiBone system is based on our previous AIBOcom architecture [3]. We hypothesise that the robot (a Sony AIBO robot in our study) will help to maintain and strengthen participants' distanced relationship [4]. AiBone controls two AIBOs, each interacting with a remote participant, providing the ability to play a 2-player game via the AIBOs. The human-robot interactions were designed and implemented bearing in mind both robot and human perspectives for robot "sociability" (cf. [5, 6]). In this game, participants were required to guide their virtual characters through various game levels in order to find randomly hidden rewards ("bones" in our dog-inspired scenario, Figure 1) using the AIBOs as an interaction tool. Importantly, and different from conventional haptic interfaces, each robot is an autonomous system with its own set of behaviours, sensing abilities, and internal variables ("needs", cf. [7, 8]) and the participant is expected to interact with the AIBO like a real pet in order to regulate the dynamics of the interaction. During the task, the design of which has been inspired by the theme of dogs trying to find bones, the AIBOs autonomously execute dog-like behaviours (cf. $[9,10])$ that are controlled by the robot's internal variables. The task offers to the participants the option to co-operate and receive double points for each hidden bone they find in cooperation. Alternatively, they can search for the bones individually, either by negotiating to split the area among themselves or by searching randomly and independently. AiBone also offers the option to complete the task by using conventional input devices and this allows us to compare participants' experience associated with the use of robot.

The main research question of our study was:

How do participants perceive, react to and behave in a collaborative scenario involving remote human-human interaction using robots as social mediators?

In this study we developed the AiBone platform in an attempt to evaluate participants' interactions with the robot and additionally, to explore human-human and human-robot behaviours and record any effects arising as a result of the occurring social stimuli during the communication. In order to identify and evaluate the effects of robots in such a context, we introduced as a baseline an experimental condition where conventional interfaces replaced the robots. Initially, it was anticipated that participants would engage and express more social behaviours derived from the presence of robots when utilising AIBOs as social mediator compared to using conventional interfaces. Additionally, we expected participants to enjoy the human-robot interactions considering that the robot offers various expressive behaviours linked to specific game events and is consequently expected to make the game experience more interactive and engaging in contrast to a communication mode without robots.

The paper is organised as follows. Related research studies that focus on Human-Robot Interaction (HRI) and human-robot engagement are discussed in section 2. Section 3 describes the AiBone system and section 4 the experimental setup. Section 5 presents experimental and statistical results. Section 6 discusses and analyses the results and lastly this paper ends with the Conclusions section.

\section{Related research}

The "Huggy Pajama" is an example of similar previous research in the field of $\mathrm{HRl}$ which also combines remote communication and robotics [11]. The project comprises a special pyjama that is worn by a child and a huggable robotic bear. While a parent is away from their child, they can hug the robotic bear that has been equipped with pressure sensors. The hug is transmitted to the child via inflatable pads in the worn Pajama. The related "Hug over distance" system is identical to Huggy Pajama and uses the same hardware to simulate the distant hug, with the only difference being that it focuses on couples rather than parent to child interaction [12]. Evidently, both aforementioned systems require the use of special hardware, which may be difficult to acquire and may be intolerable by some users, especially children. In addition, a user is required to wear the special vest continually in order to sense the distant hug, which might prove to be impractical or unacceptable for long-term use. Also, hugging is a very specific form of social interaction and limited in its application to wider scenarios involving not only close friends or family members.

MIT's "Huggable" is a similar project that has specifically intended the enhancement of remote communication between parents and their children [13]. The Huggable hardware is remotely controlled via a specifically designed web interface. The particular robot is programmed to run in a semi-autonomous mode which allows it to react to various externa stimuli. A possible usage for such a system could be cases where a parent is away and he or she could use the web interface to connect to the robot bear and use it to read their child a story.

Furthermore, "MeBot" is a telepresence robot that allows the users to express non-verbal behaviours that are commonly used in face-to-face interactions [14]. The robot consists of a small moveable body with servo operated hands that perform teleoperated gestures and movements. A small-sized screen is integrated in the robot's head and displays the other user's face. Results of user studies showed that in a comparative study, users preferred the MeBot communication over the static robot that could not move and express behaviours. Furthermore, users found the expressive robotic embodiment more engaging and enjoyable and it was concluded that the option of remote expressiveness contributed to a better cooperation. Similar to MeBot, but with less complicated hardware, is the "GestureMan" mobile robot [15]. GestureMan was mainly developed in order to embody a remote operator's instructions by using a Pioneer base unit as the mobility gear and a camera with tilt ability and a laser pointer to express and capture human behaviours. The operator controlled the robot with the help of a remote joystick and a wireless camera. With the help of GestureMan, the researchers showed that a remotely controlled robot similar to their design has the ability to embody the actions of the remote instructor.

The above mentioned projects have shown that touch and teleoperated gestures can enhance interactions between humans and robots, even in cases where robots are located away from their operators. Further to these projects, a much related research area is that of haptic technologies. Haptics is used in systems in order to provide touch feedback to the operators/users [16]. The majority of computer games nowadays are typically limited to visual and audio perception with hardly any touch input. One of the reasons is that the development of computer games that offer haptic experience to gamers (and users with special needs, i.e. visual or audio impairments) can be challenging [17]. To give an example of research in this area, "HaptiCast" is a game test bed which allows the users to experience the feeling of touch and increases the level of immersion and entertainment through haptic interactions and effects in 3D games [18]. In this particular project the users are utilising 


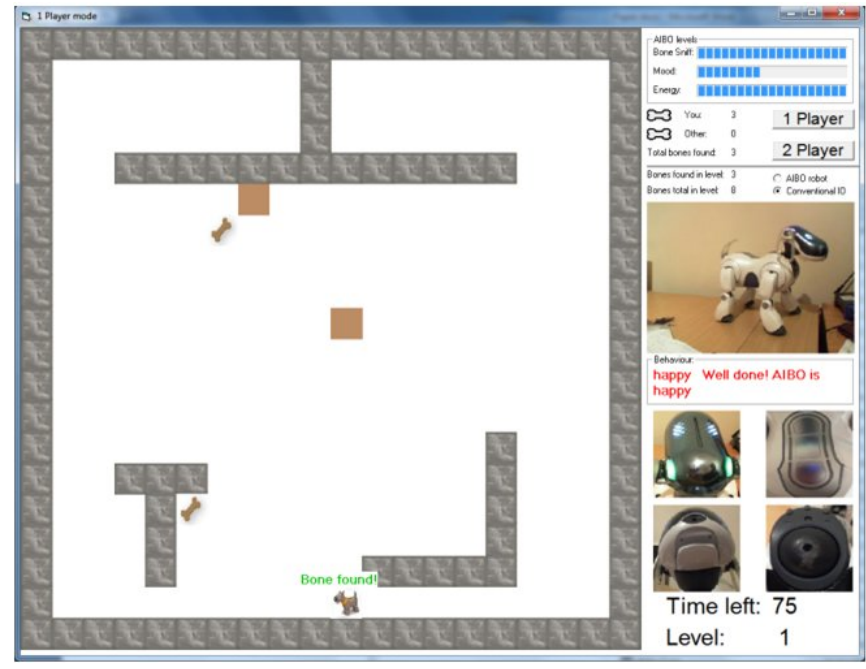

Figure 1. AiBone game interface in Conventional mode.

a PHANTOM $^{7}$ haptic device in order to move and interact inside a 3D virtual world. Another related project is the "Haptic Battle Pong" where two users play the traditional Pong game, with the only difference being that the game is in 3D, and instead of a keyboard or joystick users are utilising a PHANTOM haptic device to control their characters [19]. Research indicated that users preferred the haptic device to control the game over a typical conventional computer interface. It is worth stressing the point that users found the game intriguing and effective as their sense of interaction was substantially enhanced in a two player mode compared to an ordinary computer game. On the other hand, not only do specially made haptic devices offer the sensation of touch, but commercial joystick interfaces provide a similar experience with motor vibrators. Through the use of joysticks, users can also sense and visualise the virtual game structures [20]. The principal advantage of using these devices is that they cost only a fraction of a special haptic device's cost (such as the PHANTOM) while at the same time they offer force feedback which can be very useful in providing visual information to users with sensory impairments. However due to the straightforwardness and simplicity of commercial joystick interfaces, only simple representations and shapes can be visualised to the users. The above mentioned research investigates the role of haptic devices on remote communications that are able to provide feedback to both users, however, little has been done on utilising commercial entertainment robots in order to provide the same effect to the users, which is the key approach of this article.

\section{System overview: AiBone}

The AiBone software allows two robots to be connected to a computer and handles the synchronisation between robots and participants. The

7 Phantom Haptic device:

haptic-phantom-omni.htm
Table 1. Mood variable affect values from the game buttons

\begin{tabular}{|l|l|l|l|}
\hline & $\begin{array}{l}\text { Head } \\
\text { Button }\end{array}$ & $\begin{array}{l}\text { Back } \\
\text { Button }\end{array}$ & $\begin{array}{l}\text { Chin } \\
\text { Button }\end{array}$ \\
\hline $\begin{array}{l}\text { Mood value } \\
\text { (Max 30) }\end{array}$ & +5 & +2 & +8 \\
\hline
\end{tabular}

Table 2. AIBO internal states affecting values from various active behaviours.

\begin{tabular}{|l|l|l|l|l|l|}
\hline Frequency & \multicolumn{5}{|l|}{ Every 1.5 seconds } \\
\hline $\begin{array}{l}\text { Active } \\
\text { Behaviour }\end{array}$ & Bored & Tired & Digging & $\begin{array}{l}\text { Ball } \\
\text { Visible }\end{array}$ & $\begin{array}{l}\text { Ball Not } \\
\text { Visible }\end{array}$ \\
\hline $\begin{array}{l}\text { Mood Value } \\
(\max 30)\end{array}$ & +1 & 0 & 0 & 0 & -1 \\
\hline $\begin{array}{l}\text { Energy Value } \\
(\max 30)\end{array}$ & +1 & +3 & -3 & 0 & 0 \\
\hline
\end{tabular}

software consists of the interface which is a 2D maze game and the robot software that controls the robots. The robot control software is written in URBI [21], a universal language that supports various robots, and that makes AIBOcom [3] and AiBone a multi-robot platform. The main goal of the maze game is to guide a virtual dog-like character around various levels and find as many hidden bones as possible. The guidance of the virtual character can be done in two ways; either by using keyboard and mouse (Conventional mode) or by using the robot as an autonomous robotic helper (Robot mode).

In the Conventional mode the arrow keys control the character in the maze and the mouse helps the participant to cope with the deficit of various internal states (Mood and Energy) of the character by clicking on specific images [Figure 1 - bottom right]. Each picture affects both "energy" and "mood" values of the robot depending on the area that the participant clicks with the mouse (Table 1) (e.g. the chin button increases the mood value significantly compared to a smaller increase after selecting the head or back button).

During the game, participants have to utilise the internal state values in order to efficiently guide their virtual character. The energy value affects the speed of the character and when the value reaches a certain threshold, the character freezes until it regains its energy. The mood value freezes the character as well while the participants can cope with this by interacting with the provided buttons that affect the mood value. These buttons along with the associated animated AIBO picture, offer the participant a virtual representation of an AIBO robot allowing him to affect its virtual internal states similarly to those of the physical robot. In the Robot mode, the control of the virtual character's movement is realised by real-time tracking of a pink ball moved by the participant in front of the AIBO's visual field. We preferred to use this method of interaction for the virtual character navigation in order to simulate a real dog ball game and to reduce the participant's cognitive load during the human-robot interaction. As the AIBO robot moves its head to follow the ball, the character on the screen moves horizontally or vertically inside the maze accordingly. An example of a movement is when the participant wants to move his virtual character left therefore, he will need to drag the provided pink ball towards the left side of the AIBO in order to follow it. During the game, while the virtual character is wandering in the maze controlled by the participant, the robot performs various sniffing behaviours when it gets close to a hidden bone in order to help the participant identify the exact location. As the goal of this game 
Table 3. Demographic data.

\begin{tabular}{|l|l|l|l|l|l|l|l|l|l|l|l|l|l|l|l|l|l|l|l|l|}
\hline Pair & 1 & 2 & 3 & 4 & 5 & 6 & 7 & 8 & 9 & 10 & 11 & 12 & 13 & 14 & 15 & 16 & 17 & 18 & 19 & 20 \\
\hline Age (mean) & 27.5 & 43.5 & 27.5 & 24.5 & 29.5 & 25.5 & 28 & 26 & 23 & 25.5 & 23 & 27.5 & 31.5 & 19.5 & 21 & 19 & 19 & 23 & 21.5 & 36 \\
\hline Relationship & $\mathrm{C}$ & $\mathrm{C}$ & $\mathrm{F}$ & $\mathrm{F}$ & $\mathrm{F}$ & $\mathrm{F}$ & $\mathrm{F}$ & $\mathrm{F}$ & $\mathrm{F}$ & $\mathrm{F}$ & $\mathrm{F}$ & $\mathrm{F}$ & $\mathrm{C}$ & $\mathrm{F}$ & $\mathrm{F}$ & $\mathrm{F}$ & $\mathrm{C}$ & $\mathrm{F}$ & $\mathrm{F}$ & $\mathrm{N}$ \\
\hline $\begin{array}{l}\text { Familiar with } \\
\text { robots (mean) }\end{array}$ & 1.5 & 3.5 & 3.5 & 2 & 1.25 & 1.5 & 2 & 1.5 & 2.5 & 3 & 2.5 & 2 & 4 & 2 & 2.5 & 2.5 & 3.5 & 2 & 3 & 2 \\
\hline $\begin{array}{l}\text { Familiar with } \\
\text { computer Games } \\
\text { (mean) }\end{array}$ & 2 & 3 & 3.5 & 4.5 & 3.25 & 4 & 2 & 3.25 & 5 & 4 & 3 & 3.5 & 5 & 4.5 & 4 & 5 & 5 & 4 & 3.5 & 2.5 \\
\hline $\begin{array}{l}\text { Video } \\
\text { communication } \\
\text { experience (mean) }\end{array}$ & 1.5 & 1 & 2 & 5 & 5.5 & 6 & 2.5 & 7.5 & 2.5 & 15 & 10.5 & 4.5 & 11.5 & 6.5 & 3 & 9.5 & 1 & 5.5 & 3 & 1.5 \\
\hline
\end{tabular}

(Age: Mean values, Relationship: $C=$ colleagues $-F=$ Friends $-N=$ None, Familiarization values indicate 1 for low and 5 for maximum, Communication experience shows the average weekly hours participants spent on video communications)

is to find as many bones as possible, participants were motivated to search each maze level thoroughly. When the virtual character is in the radius of 2 game blocks from the hidden bone the robot automatically performs one of the 5 different behaviours depending on its relative position to the virtual bone on the game and while it is executing them, it provides physical feedback to the participant in order to help them locate the bones more efficiently. Therefore the behaviours physically direct the participant to guide the virtual character closer to the bone by pointing in the right direction. An example of such behaviour in a game arena with $20 \times 20$ game blocks (see Figure 1) is when a hidden bone is located 2 blocks away from the left side of the virtual character and AIBO physically points towards the left with its head while it is moving the left ear and playing back a sniffing sound.

Similarly to the conventional mode, participants have to keep the Energy and Mood values in sufficient levels in order to successfully wander in the game area and find the hidden bones in the given time. However, in the robot mode the various robotic images that allowed the participant to modify the internal states are not available any more. In this mode, AIBO robots are linked directly with the software and mirroring their internal states with the system. Consequently, every human-robot interaction (e.g. petting) and the various AIBO behaviours affect the Energy and Mood level in accordance with the values of Table 2. The lower threshold for both Mood and Energy values have been set to 8 and the upper threshold to 27 for this study. Whenever the level of the Mood or the Energy reaches the lower value, AIBO executes the boredom or the tiredness behaviour consequently. The Mood and Energy levels are linked with factors such as Time, user interaction (e.g. petting or ball tracking) and active behaviour.

Each level has a time limit of 75 seconds (selected in pre-trials as an appropriate time interval) and during this period the participants have to find the hidden bones that are randomly distributed in the maze. For every game level, a different bone distribution pattern was used (the same for all participants) in order to increase the difficulty level proportionally to the game level. The game interface also includes 3 horizontal graphic bars to display other useful information to the participant such as the virtual character's energy and mood (internal states) and the sniffing value. The sniffing bar displays the virtual character's relative distance from the bone and it reaches the maximum level when the virtual character stands directly on the bone location. The bar works similarly to the AIBO sniffing behaviour as mentioned above, however, it is limited only to visual information (i.e. sniffing bar value) to inform the participant about the proximity of the hidden bones. The sniffing bar is active and displays information about the hidden bones only in the Conventional mode. The main goal of the game is to find as many bones as possible within the time limit and through different levels. Importantly, the participant can earn two points when a bone is found as a result of cooperation with the peer. In order to collaborate, both virtual characters have to be close to each other whenever they dig for the bone. Both participants will receive two points only if their virtual characters stay close to each other until the bone is being excavated. Overall, if participants want to be efficient at each level, cooperation is highly encouraged to cover every spot of the maze and thus maximise the score in the limited time. Each participant can also see the remote participant's robot's internal states on top of their character like in an ordinary $\mathrm{RPG}^{8}$ game in order to coordinate their movements and actions and achieve their goal of scoring high in each level more efficiently.

Generally, the Robot mode will allow the participants to share the robots' interactions and collaborate in order to achieve a common task. We designed the robot mode to mirror continuously the internal states therefore, the system can enhance users' perception of interacting together with a same robotic platform. Additionally, the presence of robots on both sides will allow the participants to exhibit more social cues with each other as the dog-like behaviours we designed offer an enjoyable human-robot interaction.

\section{Experiment}

In this section we describe the experiment setup along with the methods used to record and investigate the participants' performance along with their interactions with the robot.

\subsection{Participants}

For this study we recruited 40 male participants (20 pairs) aged between 18 and 53 and paired them randomly. We did not intend to investigate gender differences in this exploratory study, therefore, we

8 RPG stands for Role Playing Game where users control small virtual characters on the screen and for every character a small bar is displayed on top of it showing information such as health, energy etc. By observing these bars, the user can coordinate and control its players (virtual characters) more efficiently. 
used only male participants in our experiment. Most of the participants were University students while others were academic staff from various disciplines. Table 3 displays the demographic details for each pair. The demographic data, including their relationship with the remote participant, were taken prior the study from the participants.

\subsection{Procedure and experimental setup}

Each participant was seated in front of a computer with an AIBO robot, and then started communicating with the other participant located in a separate room using a combination of webcam and microphone through Internet conference software (i.e. Skype). Participants were given approximately 5 minutes for each playing mode in order to familiarise themselves with the AiBone software (for each mode of interaction), and then continued with the study unless they needed more practice time in a specific mode. In order to consider the familiarisation factor for a specific mode, we counterbalanced the order of the two playing modes.

\subsection{Data collection}

Each room was equipped with a second video camera, used to capture the behaviour of the participant, the robot and their interaction. In addition, AiBone recorded a series of log files which contained all participant input and game states such as the virtual character's position, local and remote AIBO internal states, game mode, date and time. Furthermore, each AIBO stores an internal log file into its memory card as it captures every event (e.g. petting history, ball visibility, executed behaviours etc.) with timestamps in order to analyse the human-robot petting history and robotic behaviours. Both log files have been used in our statistical analysis after they were extracted and imported into Microsoft Excel. At the end of each run, participants were given a questionnaire to fill in, which allows us to assess their game experience.

\subsection{Methods}

For the video data analysis we used Noldus Observer $\mathrm{XT}^{9}$ to analyse and code participant's behaviours. We used the following coding scheme for the videos:

i) Gaze

Gaze at screen

Gaze at robot

ii) Smiles

iii) Talk

Socially Oriented

Speech to other participant about AIBO experience

Speech to other participant about something else

\section{Task Oriented}

Negative comments about AIBO

9 Noldus Observer XT behavioural coding suite (2009) http://www.noldus.com/human-behavior-research/products/ the-observer-xt, Accessed Mar 25, 2011

\section{Positive comments about AIBO \\ Speech to the other participant about game}

The above coding scheme was used to statistically analyse the affects of participant gaze, smiles and speech relatively to participant enjoyment and preference (questionnaire data) during the game in both modes. From the video analysis we chose behaviours that characterise human-robot and human-human interactions. The gaze behaviour was chosen in order to evaluate the participants' perception on the game and generally on the proposed platform. This information reflects the time that each participant spent looking at the robot while playing the game and indicates how focussed and concentrated the participant was. Besides gaze, since smiles typically indicate a positive social cue towards the game and the remote participant, we also count the number of smiles shown by each participant in each gaming mode. We assume that smiling during the game reflects the participant's enjoyment while playing the game. Furthermore, according to Slattery [23] smiles are considered a very strong social cue between humans in social interactions. Along with the smiles and gaze, we also coded speech and its nature (socially or task oriented) in order to measure the exchanged verbal social cues between the participants. The socially oriented speech was divided into 2 further categories, representing the number of times the participant talked to the other participant about his AIBO or about something else. Task oriented speech is coded when a participant talks about the game task while socially oriented is when he talks about nongame related matters. An example of task oriented speech is when the participants coordinate their movements in the virtual environment via speech in order to succeed in their task or comment about specific AIBO features and capabilities (e.g. "AIBO is too slow", "AIBO helped me finding the bone" etc). The task oriented speech was further divided into 2 categories, measuring the participant's positive or negative comments about his robot which are directly related to the task, and speech about the game which most frequently concerned cooperation in the game. Finally, further to the video analysis and questionnaire data, the robot's internal log files and the game log files have been used to extract information regarding the percentages of petting and ball visibility during the game.

\subsection{Data analysis}

Results from the questionnaire data were statistically analysed. The questionnaire data shows the median value of participant experience of cooperation and coordination between the participants in Conventional and Robot modes respectively, as well as the median value of the overall game experience on both modes. From the game log files we extracted the collected "bone values" (rewards) for each level and we also distinguished the collection method (individual or co-operative collection). Individual collection occurs when one virtual character finds and collects the bones on its own, whereby co-operative collection occurs when both participants cooperate to find and collect the same bones and are thus getting double points as described above in section 3. By using this information we can assess the participants' performance for each level and the degree of cooperation for both playing modes. Concerning the data collected from the robots we analysed the log files which captured the number of petting events from the participants and the percentage of ball visibility. We analysed the "petting value" as it is important to assess the interaction frequency between the participant and the robot. Since the robot runs in autonomous mode and executes certain behaviours, it requires feedback and input from the participant. The "visibility value" derives from the AIBO camera that tracks the provided pink ball and records the number of occurrences when the ball was visible for each game. It shows the participant's performance in 
regards to controlling the $\mathrm{AIBO}$ in a sufficient way, i.e. understanding the concept of controlling and interacting with the robot.

In order to evaluate the video coding reliability, a trained secondary observer coded $20 \%$ of the videos (8 randomly selected videos out of 40 for both game modes) by using the same procedure as the original observer. The observations of the secondary observer were compared with the original observations using Cohen's Kappa statistics [24] in order to measure the inter-rater agreement $[25,26]$.

\section{Results}

Firstly, we discuss results from the inter-rater agreement tests. Figures 2, 3 and 4 show the human-robot interaction derived from the video data and the robots' internal log files. Next, in Figure 5, we present the participants' questionnaire results regarding cooperation and coordination between the participants and their overall experience of the game. Figures 6,7 and 8 summarise the social effects of an autonomous robot on human-human communication during their interactions with the AiBone system. Finally, Figures 9 and 10 present the game scores that each participant managed to achieve during the game.

\subsection{Inter-rater agreement}

Table 4 shows the detailed inter-rater Kappa values. The values of the table show the number of agreements and disagreements between rater 1 and 2 along with the proportion and the Cohen's kappa coefficient value in order to check the reliability of the video coding. At the bottom of the table average values are shown for each column. The Kappa values range between 0 and 1 and a value close to 1 indicates better reliability. Many researchers use the proposed Fleiss scale in order to interpret their reliability results where $0.40-0.60$ values are understood as fair agreement, $0.60-0.75$ as good agreement and values greater than 0.75 as excellent [27]. A Kappa value of greater than 0.60 is considered sufficient to ensure that chance by itself is not likely to have an effect on the agreement. The reliability analysis was performed with the use of a tolerance window of 1 second and aligned the observation times for accurate synchronisation. The Robot mode coding produced lower Kappa values between the inter-raters because in these videos participants also interacted with the AlBO besides the computer, and therefore, gazing on different objects was included in the coding and it produced a large number of time based events which were difficult to code accurately and precisely from both inter-raters (e.g. multiple gaze timeline events coded with millisecond accuracy). Results show that overall the inter-rater agreement is acceptable.

\subsection{Results}

\subsubsection{Number of participants' smiles for both game} modes

Among a variety of social cues, the smiles between the participants have been recorded, see Figure 2. A Paired Samples T-Test confirmed that participants smiled more often in the Robot mode than in Conventional mode (Std Dev. $=3.282, \mathrm{t}=-4.866$, Sig. $=0.0001)$.

5.2.2. Non-task related speech between the participants

Further to the smiles, which indicate social cues, speech between the participants (Figure 3) with non-task content was also coded. In the Robot mode, participants talked more frequently (37 times compared
Table 4. Inter-rater agreement and Kappa values.

\begin{tabular}{|l|c|c|}
\hline Participant - Mode & Proportion of agreements & Kappa value \\
\hline 14 - Conventional mode & 0.78 & 0.62 \\
\hline 14 - Robot mode & 0.72 & 0.55 \\
\hline 17 - Conventional mode & 0.81 & 0.71 \\
\hline 17 - Robot mode & 0.76 & 0.64 \\
\hline 25 - Conventional mode & 0.94 & 0.78 \\
\hline 25 - Robot mode & 0.73 & 0.54 \\
\hline 26 - Conventional mode & 0.93 & 0.73 \\
\hline 26 - Robot mode & 0.71 & 0.54 \\
\hline 35 - Conventional mode & 0.92 & 0.79 \\
\hline 35 - Robot mode & 0.72 & 0.64 \\
\hline 37 - Conventional mode & 0.78 & 0.66 \\
\hline 37 - Robot mode & 0.79 & 0.62 \\
\hline 40 - Conventional mode & 0.91 & 0.64 \\
\hline 40 - Robot mode & 0.74 & 0.62 \\
\hline Average & 0.80 & 0.65 \\
\hline
\end{tabular}

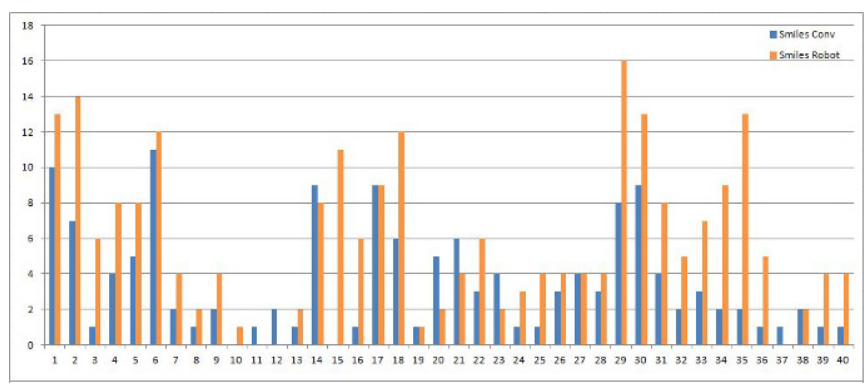

Figure 2. Participants' smiles for Conventional and Robot mode.

to 2 in the Conventional mode) about non-game related matters as well as sharing their experiences of the AIBO robot.

\subsubsection{Task-related speech}

Figure 4 represents how often participants spoke to each other about game related matters. A Paired samples T-Test has been performed in order to validate our assumption that participants talked more in the Conventional mode than in the Robot mode and the results are significant (Std Dev. $=11.73, t=5.107$, Sig. $=0.0001$ ).

\subsubsection{Participants' experiences in the two playing} modes

Figure 5 presents the participants' experiences in the two playing modes:

The overall game experience indicates the same participant preference, based on median questionnaire values, for Conventional and Robot modes. On the other hand, the cooperation and coordination preference between the participants is significantly lower in the Robot mode as calculated by using Wilcoxon's statistical test $(Z=-4.802$, Sig. $=0.0001)$. 


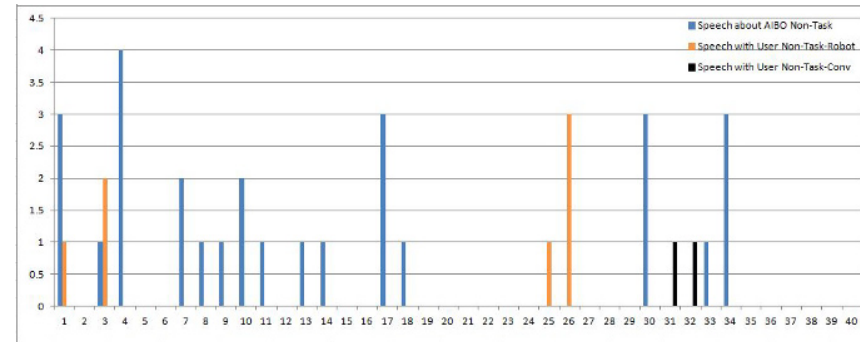

Figure 3. Speech with non-task content.

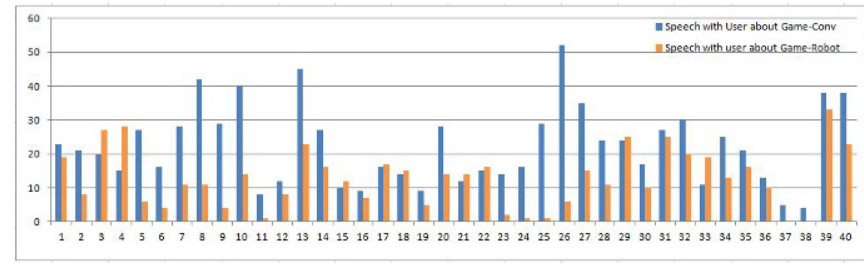

Figure 4. Task-related speech between the participants for both playing modes.

\subsubsection{Gaze at AIBO (percentages)}

Figure 6 presents the total percentage of gaze at robot during the robot game mode:

Participants spent on average $18.21 \%$ of their time looking at the robot while playing the game. (Std Dev. $=7.72$, Mean=18.2, $M i n=2.89$, $\operatorname{Max}=32.04)$.

\subsubsection{AlBO ball visibility}

Figure 7 displays the percentages of time when AIBO managed to track the pink ball during each game. The data is derived from the robot's internal log files and is statistically analysed resulting in a mean value of 76.32\% (Std Dev.=7.34, Min= 65.5, Max=94).

\subsubsection{Number of robot petting behaviours}

Figure 8 shows how often participants petted their robot dogs on the head or on the back sensors. This information has been extracted from the robot's internal log files and has been statistically analysed with a

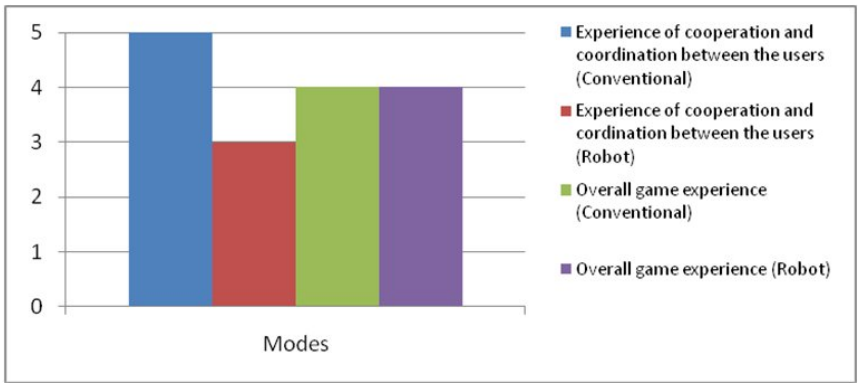

Figure 5. Results of questionnaire data for participant experience in both modes.

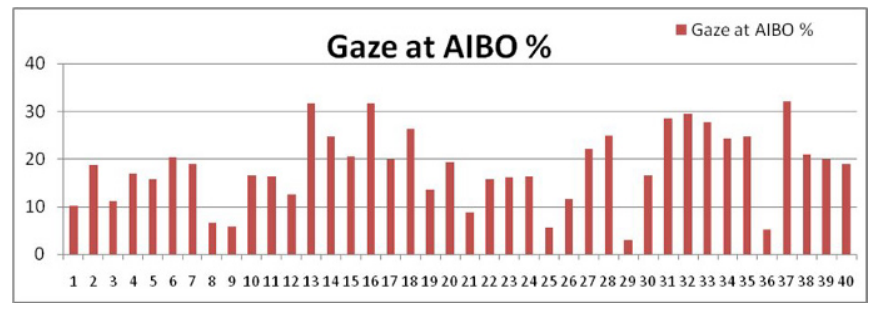

Figure 6. Participant gaze at AIBO in percentages.

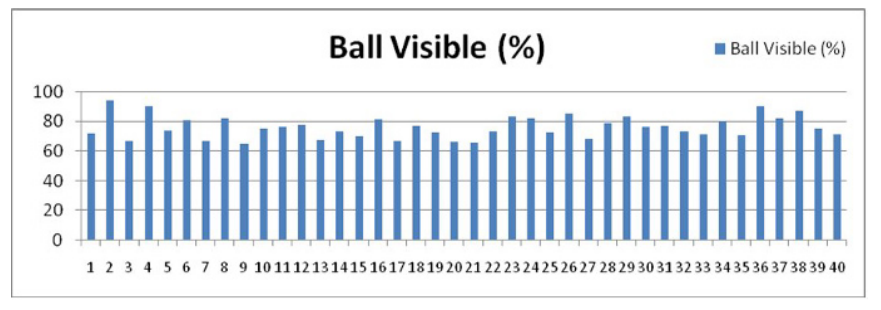

Figure 7. AIBO ball visibility percentages for each participant.

mean value of 57.95 (Std Dev. $=39.1, \mathrm{Min}=4, \mathrm{Max}=142)$.

\subsubsection{Total collected bones for both modes}

The last two graphs (Figures 9 and 10) show the scores of total collected bones by each participant in the two playing modes.

In Figure 9 it is clearly shown that participants managed to collect more bones in the Conventional mode than in the Robot mode and the results are significant as shown after a Paired Samples T-Test (Std Dev.= 3.142, $t=25.863$, Sig. $=0.0001$ ). Furthermore, Figure 10 shows the differences between the collected double points (in cases where they cooperated) between the participants, with statistically significantly higher scores for the Conventional mode (Std Dev. $=1.657, \mathrm{t}=12.785$, Sig. $=$ 0.0001). There is also a high degree of correlation between the two modes, whereby participants who managed to get high scores in one mode also got a high score in the other mode. A Pearson's correlation statistical test was conducted on the above results for both 'Total' and 'Double' collected points respectively and revealed a proportional significant relation among them (Total points: Pearson correlation $=0.933$, Sig. (2-tailed) $=0.0001$, Sig. at level $=0.01$ and Double Points: Pearson correlation $=0.701$, Sig. $(2$-tailed $)=0.0001$, Sig. at level=0.01) .

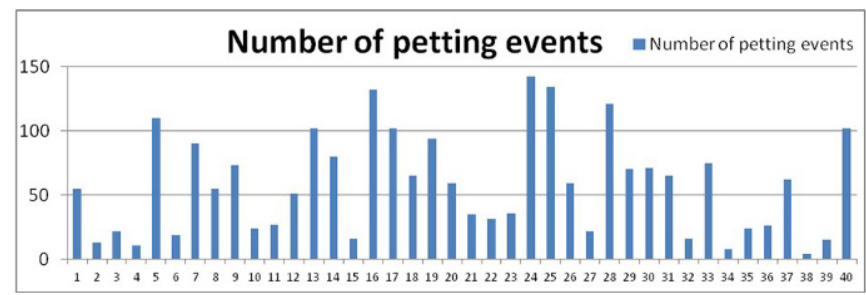

Figure 8. Number of petting the robot of each participant in the Robot mode. 


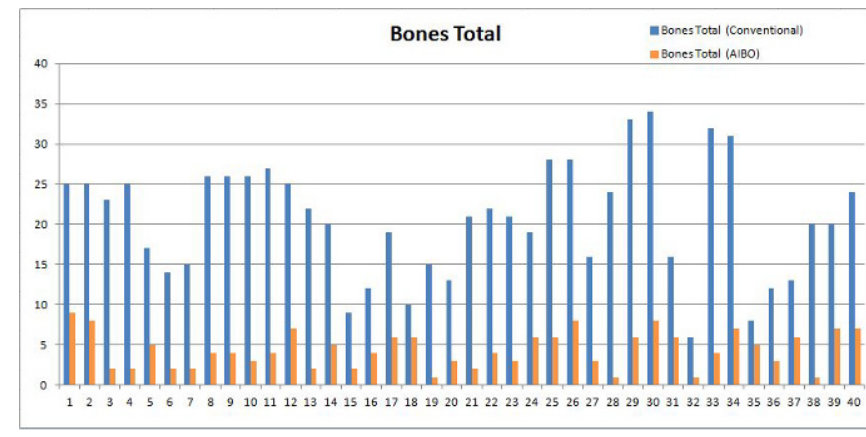

Figure 9. Total number of bones found for both playing modes.

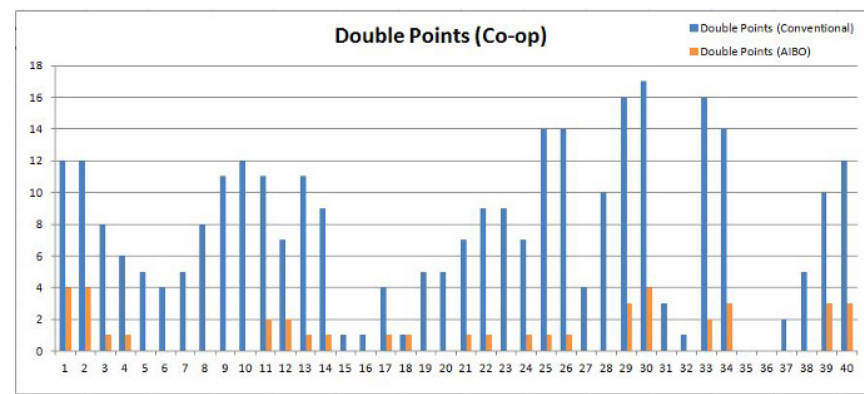

Figure 10. Total of double points gained from cooperation in both playing modes.

\section{Discussion}

The main focus of this study was to analyse participants' experience and preferences in a cooperative two-player game involving robots in the role of social mediators. Further to this question, we wanted to study the social influence of our system on the participants while communicating and playing the game remotely, compared to a setup using only conventional, non-robot interfaces.

Figure 2 showed the number of times participants smiled at each other while playing the game in both modes. Smiling provides a positive social cue to the other participant and it is usually perceived as an indication of enjoyment while the participants communicate visually. In the Robot mode, participants might have got more excited when they collected points because it was more demanding than the Conventional mode, and thus they were more satisfied. The recorded smiles for both modes and clearly show that participants smiled almost twice as much in the Robot mode compared to the Conventional mode. Concerning speech, Figure 3 clearly showed that participants talked more frequently about non-game related matters as well as sharing their experiences about the AIBO robot, compared to the Conventional mode. The result that not every participant talked about non-game related matters might have been caused by the fact that the game was designed with a strong focus on task achievement. Further to this, the time limit for each level complicated the task and stressed the participants to find the bones in time - thus not leaving them many opportunities to socialise through the game. Figure 4 presented the task related speech where participants generally spoke with the same rate in both modes with no significant fluctuations on most occasions. On average, in the Conven- tional mode participants talked more than in the Robot mode regarding task related matters, probably because the difficulty of controlling the virtual character in the latter mode discouraged the participants to cooperatively search for bones within the time limit for each level, and therefore, they sought for the bones individually (resulting in less task related conversations).

Figures 6, 7 and 8 displayed the interaction patterns between the participants and the robots. Figure 6 showed the percentage of gaze at the computer screen in the Robot mode which has been extracted from the video observations. The mean gaze value for the participants is $18 \%$ which shows how little time they spent looking at the AIBO during the game in the Robot mode which is comparatively lower than the gaze at the screen. This observation suggests that participants found it confusing to focus on 2 different objects while performing a game oriented task on the computer. The limited gaze on the robot may affect participant's perception regarding robotic behaviours and robot control as required by the game. Thus, it might have been difficult for the participants to fully understand the principles of human-robot interaction afforded by the game. This argument can be supported by results from the robot's internal ball tracking log files. This log file captured the number of times that the pink control ball was in sight, thus allowing the robot to control the game. Figure 7 pictured the number of times that AIBO successfully tracked the provided pink ball during the game in percentage values. The average ball visibility is $70 \%$ during the game which is generally low for sufficient game control since the primary input to the robot is the provided pink ball. Theoretically, ball visibility should be very close to $100 \%$ for continuous virtual character control like in the Conventional mode where participants persistently press the keyboard/mouse buttons. Figure 8 depicted the number of times participants petted their AIBOs during the game. The number of robot petting was also quite low which led to poor game performance because of the continuous triggering of the robotic boredom behaviour - the AIBO was running in autonomous mode which requires participant attention whenever the boredom internal state drops. On every execution of the boredom behaviour of the robot prevented the participant from continuing to play for a certain amount of time until the participant 'satisfied' the robot's internal states. The robot's autonomous behaviour execution did not seem very suitable for such games which require participants to act fast and efficient (rather than 'social') in order to achieve better game performance.

Based on the questionnaire data regarding the experience of the cooperation and coordination between the participants, it is shown (Figure 5) that participants preferred the Conventional mode for the given collaborative task. It appears that the participants needed more time to familiarise themselves with the system, in particular the Robot mode, although they were given approximately 5 minutes prior to each gaming mode. An autonomous robot such as the AIBO is considered a novel and complicated device from the participants' perspectives for applications that require immediate control and coordination through an interface, as confirmed by participants' comments on their interaction with the AIBO. Comments included: "I liked the novelty of using a robot to play the game" (participant 3), "It was interesting to use a different interface" (participant 21), "It was more interesting than a traditional keyboard" (participant 16), "I was too busy trying to control robot to interact with the other participant" (participant 9), "very interesting!" (participant 7), "robot was hard to control and focus on the screen. Difficult" (participant 5). Participants found it hard to control the virtual character in time in the Robot mode, and as a result the human-human game coordination was difficult and insufficient. The decrease in taskrelated performance of the participants in the Robot mode could be explained by the increased cognitive load and higher demands on information processing involved in operating the AiBone system in the Robot mode $[28,29]$ 
Furthermore, the pre-defined time limit of each game level seem to have encouraged the participants to search for bones individually and competitively, and for that reason participants preferred the conventional keyboard and mouse input devices which are easy to use and they were very familiar with, and thus allowed them to perform the task quicker. On the other hand, the overall game experience values indicate that the participants showed the same preference for both Conventional and Robot modes. This value represents their overall experience after they finish the game in both modes including their interactions with the robot. Consequently, even though the participants found the control of the virtual character more challenging, and maybe sometimes annoying, in the Robot mode, the robotic behaviours, their interactions with the robot and the sharing of common experiences with the robot among the participants, balanced the overall evaluation of the system by the participants. In summary, the questionnaire results indicate a preference towards the Conventional mode regarding the control of the interface and human-human cooperation in the computer game, however, the presence of a robot positively affected the overall remote communication experience during the game. A robot appearance such as an AIBO (zoomorphic shape) might have had a novelty effect on the participants, therefore, further investigation has to be done in order to verify the results. Long-term studies would also be able to shed further light on these issues.

Further to the questionnaire data, Figures 9 and 10 showed the total number of collected bones of each participant between the two playing modes, which supports our previous argument that game oriented tasks are more difficult to achieve with robots. The conventional input devices such as the keyboard and the mouse more directly controlled the virtual character and therefore, the moves required in the computer game were performed faster, allowing participants to scan the game levels for bones more efficiently in comparison to the Robot mode. Figure 10 showed the difference between the two modes in terms of cooperatively collected bones where the Conventional mode scored significantly higher than the robotic mode. In the Robot mode, participants found it more difficult to quickly move their character in time (as stated before), and therefore, they preferred to continue looking for hidden bones individually by searching separate places on each levels. Generally, with this specific game, participants in the Robot mode showed more social cues during the game. In summary, we demonstrated that an autonomous robot can be used for remote humanhuman communication in the context of a collaborative game; but when it is compared to a Conventional mode of interaction, participants consider it less suitable to finish the given task in time. Participants are very familiar with the Conventional mode (keyboard and mouse) for many years while on the other hand, an autonomous robot is new technology to them. Also, the robot had not been designed specifically for the purpose of replacing conventional interface devices but to allow them to share human-robot experiences and increase their social bonding. However, although AIBO does not offer the same performance in such task oriented games compared to the Conventional mode, it increases the social interactions between the participants thus offering a new communicative experience to the participants. The results of this study indicate that an autonomous robot has the potential of enhancing human-human experience in remote communications and consequently make it more enjoyable when the purpose of the interaction is less competitive and task oriented and more socially oriented. Additionally, based on the significant results shown above and along with the participants' questionnaire data regarding game mode preference, it is shown that although the participants preferred the Conventional mode for playing such games, that does not mean that the participants did not actually enjoy the overall communication experience in the Robot mode. Bringing a robot to such 2-player computer games inevitably makes the game task more difficult and more effortful to the partici- pants since they have to cope with and focus on two different objects at the same time i.e. controlling the game character and providing input with the autonomous robot.

\section{Conclusions and future work}

This study focused on enhancing the remote communication between two participants with the addition of an autonomous robot as a social mediator and gained positive results in regards of social cues used in human-human communication. We added an interactive and collaborative computer game to the communication channel between the participants in order to enhance their interaction experience during the video call. In this study we analysed participants' behaviours and reactions with our system and explored their preferences during the remote communication. We analysed questionnaire data, robot and game log files and videos in order to answer our research questions and expectations. Results showed that participants found the Robot mode exciting although sometimes they would get frustrated because of the difficulty that the new technology added. However, we also provided evidence that participants expressed more social cues and shared their game experiences through the robot with each other. Additionally, results showed that it is very difficult for participants to familiarise quickly with new technologies when they can perform the same task more efficiently with conventional devices. While long-term use of new technology may increase the efficiency of how participants operate the robot, it is likely that for standard tasks such as controlling a computer game character on a screen, conventional interfaces may be superior in terms of performance. Finally, we also discussed the factors that caused participants' frustration especially for controlling the robot that need to be carefully considered and addressed when designing a human-human communication tool with robots as social mediators.

This exploratory study informed us about the human-robot and humanhuman interactions in remote communications with robots as social mediators compared with a traditional method of interaction. The findings and the participants' feedback gave encouraging evidence for using autonomous, interactive robots as social mediators for distanced communication. While the robot's dog-like appearance might have led to a novelty effect in our experiments, questionnaire results supported that AIBO robots are enjoyable and preferable from the participants. Furthermore, the autonomous level of the robots affected participants attitude towards the robots and facilitated them to express more social cues between them. For that reason, we believe that a richer repertoire of embedded dog-like behaviours and impulsive AIBO actions will enhance participants' perception of interacting with an autonomous intelligent robot thus encouraging them to share their personal experiences. Additionally, results showed the importance of selecting an appropriate medium of supportive interaction through the computer interface, in our scenario the computer game, as task oriented applications require a lot effort from the users. Consequently, based on these findings, we will use a non-task oriented application to support the human-human and human-robot interaction in our future work.

\section{Acknowledgments}

We would like to thank all the participants who participated in our experiment and all the colleagues for their useful feedback during the development and testing. This work was partially supported by the European Commission (EC) and is currently funded by the EU FP7 ICT-215554 project LIREC (Living with Robots and Interactive Companions). The authors are solely responsible for the content of this publication. It does 
not represent the opinion of the EC, and the EC is not responsible for any use that might be made of data appearing therein.

\section{References}

[1] M. Folgheraiter, G. Giuseppina, L.V. Dario, A Glove Interface with Tactile Feeling Display for Humanoid Robotics and Virtual Reality Systems, Second International Conference on Informatics in Control, Automation and Robotics (ICINCO), Barcelona, Spain, 2005

[2] T. Fong, I. Nourbakhsh, K. Dautenhahn, A Survey of Socially Interactive Robots, Robotics and Autonomous Systems, Volume 42, Issues 3-4, 2003, 143-166

[3] F. Papadopoulos, K. Dautenhahn, W.C. Ho, M.L. Walters, AIBOCOM: Designing robot enhanced human-human remote communication technology, Proceedings of the Kansei Engineering and Emotion Research International Conference 2010 (KEER2010), 2-4 March 2010, Arts et Metiers ParisTech, Paris, France. ISBN: 978-4-9905104-0-4, 2010, 671- 682.

[4] N. Bos, J. Olson, D. Gergle, G. Olson, Z. Wright, Effects of Four Computer-Mediated Communications Channels on Trust Development, Proceedings of the SIGCHI conference on Human factors in computing systems: Changing our world, changing ourselves, 2002, 135 - 140

[5] C. Breazeal, Social interactions in HRI: the robot view, Systems, Man, and Cybernetics, Part C: Applications and Reviews, IEEE Transactions, Volume: 34, Issue: 2, ISSN: 1094-6977, 2004, 181-186

[6] K. Dautenhahn, Socially Intelligent Robots: Dimensions of Human - Robot Interaction, Philosophical Transactions of the Royal Society B: Biological Sciences 362(1480). 2007, 679-804

[7] J.A. Meyer, A. Guillot, Simulation of Adaptive Behaviour in Animats: Review and Prospect, From Animals to Animals: Proceedings of the First International Conference on Simulation of Adaptive Behaviour, MA: MIT Press, 1990, 2-14

[8] O. Avila-Garcia, L. Canamero, A Comparison of Behaviour Selection Architectures Using Viability Indicators, International Workshop on Biologically-Inspired Robotics: The Legacy of W. Grey Walter, 2002, 86-93

[9] P.H. Kahn, N.G. Freier, B. Friedman, R.L. Severson, E.N. Feldman, Social And Moral Relationships with Robotic Others?, Robot and Human Interactive Communication, Roman, $13^{\text {th }}$ IEEE International Workshop, ISBN: 0-7803-8570-5, 2004, 545-550

[10] A. Kerepesi, E. Kubinyi, G.K. Jonson, M.S. Magnusson, A. Miklósi, Behavioural Comparison of Human-Animal (dog) and HumanRobot (AIBO) Interactions, Behavioural Processes, Volume 73, Issue 1, 2006, 92-99

[11] J.K.S. Teh, A.D. Cheok, Y. Choi, C.F. Lasantha, R.L. Peiris, O.N.F. Newton, Huggy Pajama: A Mobile Parent and Child Hugging Communication System, Proceedings of the $7^{\text {th }}$ international conference on Interaction design and children, Chicago, Illinois. 2008, 250-257

[12] F.F. Mueller, F. Vetere, M.R. Gibbs, J. Kjeldskov, S. Pedell, S. Howard, Hug Over Distance, Conference on Human Factors in
Computing Systems, ISBN:1-59593-002-7, 2005, 1673 - 1676

[13] W. D. Stiehl, J. K. Lee, C. Breazeal The Design of a SemiAutonomous Robot Avatar for Family Communication and Education, Proceedings of the IEEE International Symposium on Robot and Human Interactive Communication (RO-MAN), 2008

[14] S.O. Adalgeirsson, C. Breazeal, MeBot: a robotic platform for socially embodied presence, $\mathrm{HRI}$ '10 Proceeding of the $5^{\text {th }}$ ACM/IEEE international conference on Human-robot interaction, ACM New York, NY, USA, ISBN: 978-1-4244-4893-7, 2010

[15] H. Kuzuoka, S. Oyama, K. Suzuki, K. Yamazaki, M. Mitsuishi, GestureMan: A Mobile Robot that Embodies a Remote Instructor's Actions, Proceeding CSCW '00 Proceedings of the 2000 ACM conference on Computer supported cooperative work, 2000

[16] V. Hayward, O.R. Astley, M. Cruz-Hernandez, D. Grant, G. Robles De-La-Torre, Haptic interfaces and devices, The Mechanical Systems Handbook: Modeling, Measurement and Control, CRC Press, 2004

[17] D. Archambault, R. Ossmann, T. Gaudy, K. Miesenberger, Computer Games and Visually Impaired People, Information Technologies for Visually Impaired People, Upgrade, 2007

[18] S. Andrews, J. Mora, J. Lang, W.S. Lee, HaptiCast: A PhysicallyBased 3D Game with Haptic Feedback, Proceedings of The International Conference on the Future of Game Design and Technology (Future Play), 2006

[19] D. Morris, N. Joshi, K. Salisbury, Haptic battle pong: high- degreeof-freedom haptics in a multiplayer gaming environment, Experimental Gameplay Workshop at GDC, 2004

[20] A.J. Johansson, J. Linde, Using Simple Force Feedback Mechanisms to Visualize Structures by Haptics, Instrumentation and Measurement Technology Conference, Proceedings of the $16^{\text {th }}$ IEEE, 1999

[21] J. Baillie, Urbi: A universal language for robotic control. International Journal of Humanoid Robotics, 2004

[22] T. Hartmann, C. Klimmt, Gender and Computer Games: Exploring Females' Dislikes, Journal of Computer - Mediated Communication, Volume 11, Issue 4 , 2006, 910-931

[23] R.L. Slattery, What Does that Smile Mean? The Meaning of Nonverbal Behaviours in Social Interaction, Social Psychology Quarterly 65, 2002, 92-102

[24] J. Cohen, "A coefficient for agreement for nominal scales" in Education and Psychological Measurement. Vol. 20, 1960, 37-46

[25] K. Gwet, "Inter-Rater Reliability: Dependency on Trait Prevalence and Marginal Homogeneity". Statistical Methods For Inter-Rater Reliability Assessment, 2002

[26] K. Gwet, Computing inter-rater reliability and its variance in the presence of high agreement, British Journal of Mathematical and Statistical Psychology 61, 2008, 29-48

[27] J. L. Fleiss, Statistical methods for rates and proportions. $2^{\text {nd }}$ ed. (New York: John Wiley), 1981, 38-46

[28] G.A. Miller, The magic number seven plus or minus two: some limits on our capacity to process information, Psychological Review 63 (2), 1956, 81-97

[29] F. Paas, A. Renkel, J. Sweller, Cognitive Load Theory: Instructional Implications of the Interaction between Information Structures and Cognitive Architecture, Instructional Science 32, 2004, 1-8 